\title{
USO DO VIDEOGAME COMO PROPOSTA TERAPÊUTICA PARA GANHO DE FORÇA DA MÃO APÓS FRATURA DO PUNHO: RELATO DE CASO
}

\author{
B. G. KIMURA*, N. M. L. DE OLIVEIRA**, N. N. ZAGO*** e L. F. R. M. FERNANDES** \\ * Graduando em Fisioterapia, Universidade Federal do Triângulo Mineiro, Uberaba, Brasil \\ ** Docente do Departamento de Fisioterapia Aplicada, Universidade Federal do Triângulo Mineiro, \\ Uberaba, Brasil \\ *** Discente do Programa de Pós-Graduação em Educação Física, Universidade Federal do \\ Triângulo Mineiro, Uberaba, Brasil \\ e-mail: brunogkimura@gmail.com
}

\begin{abstract}
Resumo:Esse estudo teve como objetivo analisar o efeito do uso do videogame E-Link no fortalecimento dos músculos da preensão e das pinças em um paciente com fratura complexa de punho. Caso Clínico: paciente com idade de 40 anos, sexo masculino, destro, com fratura distal de rádio e ulna à esquerda e diástase rádioulnar distal. Inicialmente foram realizados técnicas para controle do edema e alongamentos para aumento da amplitude de movimento. $\mathrm{Na}$ fase do fortalecimento foram avaliadas as medidas de força instrumental, teste de 10 repetições máximas e questionários funcionais. $\mathrm{O}$ treinamento foi realizado no membro lesado, e seguindo o regime isotônico da técnica de Oxford. Foram realizadas 2 sessões semanais, com duração de 40 minutos, totalizando 17 sessões. Os resultados obtidos nesse estudo, demonstraram 1) um aumento da força de preensão palmar e das pinças no membro lesado após a realização de um protocolo de fortalecimento por meio do videogame utilizando a técnica de Oxford, e 2) uma redução da incapacidade funcional dos membros superiores. Com base nos resultados, conclui-se que o videogame foi uma ferramenta eficaz no aumento das forças de preensão e pinça para este paciente.
\end{abstract}

Palavras-chave: Fraturas do Rádio, Fraturas da Ulna,Treinamento de Resistência, Reabilitação, Fisioterapia.

\footnotetext{
Abstract:This study aimed to analyze the effect using the videogame E-link in handgrip and pinch strengthening in a patient with a complex wrist fracture. Case study: Patient with 40 years old, right-handed, with a distal radius and ulna fracture and radioulnar diastasis in left hand. Initially were realized techniques for edema control and stretching to increase range of motion, prior to strengthening using a videogame. In the strengthening phase were evaluated the instrumental force measurements, 10 maximum repetitions test and functional questionnaires. The injured side was trained, and was performed the isotonic regimes of Oxford technique. The physiotherapy strengthening consisted in 17 sessions, 2 days per week and lasting 40 minutes. The results of this study demonstrated 1) an increase in handgrip and pinch on injured side after conduction a strengthening protocol through the videogame using the
}

Oxford technique, 2) a reduction in the disability of the upper limb. Based on the results, concluded that the videogame has been an effective tool in increased handgrip and pinch forces for this patient.

Keywords:Radius Fractures, Ulna Fractures, Resistance Training, Rehabilitation, Physical Therapy Specialty.

\section{Introdução}

As fraturas do punho e da mão têm implicações diversas quando comparados a outras regiões do corpo, uma vez que comprometem a amplitude de movimento, a força muscular, a precisão e controle dos movimentos. Qualquer lesão nessa região, por mais leve que seja, ocasiona grande incapacidade funcional que pode limitar, de maneira temporária ou permanente, a realização de atividades de vida diária[1].

Segundo a Previdência Social, de 2004 a 2013 foram registrados mais de 500.000 casos de acidentes envolvendo fraturas ao nível do punho e da mão, com destaque para as fraturas de extremidade distal do rádio e da ulna, fraturas de escafoide e fraturas de dedos [2].

O comprometimento da força dos músculos flexores e extensores do punho e da mão estão entre as principais causas do aumento do tempo de afastamento do trabalho desses pacientes. Nesse contexto, a Fisioterapia dispõe de recursos tradicionais para fortalecer a musculatura, aumentar a mobilidade e a coordenação do punho e da mão [3]. Porém muitos pacientes se queixam do tratamento fisioterapêutico ser monótono, repetitivo e demorado [4].

Em vista disso, a utilização de ambientes virtuais pode ser de grande valia para o processo de reabilitação, principalmente quando ela é feita utilizando jogos específicos que respeitem as limitações de cada paciente, oferecendo ao fisioterapeuta ferramentas de análise da evolução e resultados de tratamento. Sendo assim, esse estudo teve como objetivo analisar o efeito do uso do videogame E-Link no fortalecimento dos músculos da preensão e das pinças em um paciente com fratura complexa de punho.

\section{Materiais e métodos}


Este estudo foi aprovado pelo comitê de ética em pesquisa da Universidade Federal do Triangulo Mineiro, com parecer 1825. O paciente assinou o termo de consentimento livre e esclarecido conforme a resolução n466/12 do Conselho Nacional de Saúde.

Caso Clínico - Paciente com idade de 40 anos, sexo masculino, destro,com fratura distal de rádio e ulna à esquerda com diástase rádio-ulnar distal. A fratura foi diagnosticada pelo exame clínico e por investigação radiológica e tomografia computadorizada realizada na admissão do paciente (Figura 1). A conduta médica utilizada foi o tratamento cirúrgico com placa volar rígida com dez parafusos no rádio e dois na ulna, como representado na Figura 2.

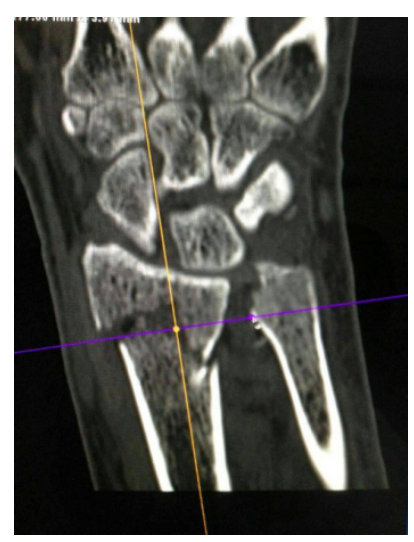

neuromuscular e alongamentos com uso da mesa canadense para aumentoamplitude de movimento do antebraço e do punho. Todas essas intervenções foram realizadas apenas no membro que apresentou a lesão.

Foram necessárias 8 sessões de fisioterapia para que o paciente apresentasse uma diminuição da dor, inicial $(E V A=8)$ em relação a final $(E V A=2)$ e um aumento da amplitude de movimento de antebraço e punho, como demonstrado na Tabela 1. Esses valores foram utilizados como critério de inclusão deste paciente para o protocolo de fortalecimento no videogame. Para iniciar o programa de fortalecimento ele deveria atingir a amplitude de movimento funcional e relatar diminuição da dor.

Tabela 1: Valores de goniometria pré e pós intervenção fisi oterapêutica
Movimentos Punho 
Medidas de ADM ativa Direito Pós

Esquerdo Pré Pré 
Figura 1: Tomografia computadorizada de punho realizado na admissão do paciente, com incidência póstero-anterior evidenciando a fratura distal de rádio e ulna e a diástase rádio-ulnar distal.

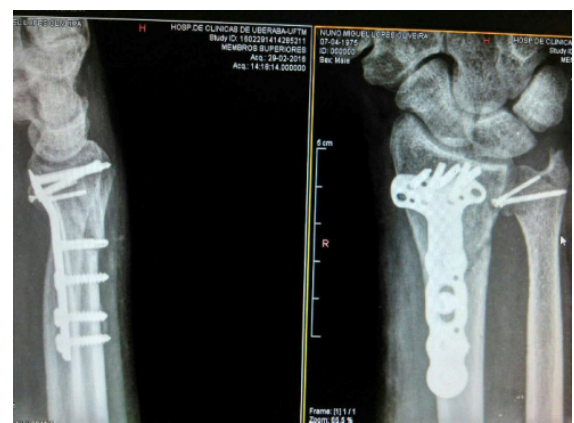

Figura 2: Radiografia de punho realizado pós-operatório nas incidências de perfil e póstero-anterior evidenciando a fixação da fratura.

O paciente iniciou a reabilitação fisioterapêutica após 30 dias de cirurgia. Previamente foi realizada uma avaliação fisioterapêutica contendo dados pessoais, Escala Visual Analógica (EVA) para mensuração da dor, goniometria de antebraço e punho para mensuração da amplitude de movimento, pré e pós intervenção.

Nessa fase inicial, o paciente recebeu orientações domiciliares diárias sobre os cuidados da pele e da cicatriz, auto alongamento, exercícios ativos e termoterapia para controle do edema. E na fisioterapia foram realizados: drenagem linfática manual para controle do edema; técnicas de inibição recíproca

\begin{tabular}{llll}
\hline Pronação & $90^{\circ}$ & 4 & $78^{\circ}$ \\
Supinação & $90^{\circ}$ & 4 & $66^{\circ}$ \\
Flexão & $90^{\circ}$ & 4 & $62^{\circ}$ \\
Extensão & $70^{\circ}$ & 4 & $56^{\circ}$ \\
Desvio & $43^{\circ}$ & 1 & $32^{\circ}$ \\
Desvio & $22^{\circ}$ & 2 & $28^{\circ}$ \\
\hline
\end{tabular}

$\mathrm{ADM}=$ amplitude de movimento ${ }^{\circ}=$ graus

Após essa fase inicial, o paciente foi submetido a uma nova reavaliação contendo medidas de força instrumental, teste de 10 repetições máximas (10RM) para cálculo das cargas de preensão e pinça para o fortalecimento e questionários funcionais, que incluem: DisabilityoftheArm, ShoulderandHand(D A S H) e PatientRatedWristEvaluation(PRWE).

Para medida das forças foi utilizado o equipamento Kit E-link da marca Biometrics ${ }^{\circledR}$ modelo H500 - Hand $k i$ t composto por goniômetros e dinamômetros de preensão e de pinça; e osoftware de avaliação para os membros superiores (EWS - UpperExtremityEvaluation Software).

No teste de força instrumental de preensão e das pinças foram realizadas 3 medidas de forçaisométricas máximas, bilaterais e calculadas a média das três repetições. Durante esta avaliação o paciente foi instruído a permanecer sentado, com o cotovelo fletido a $90^{\circ}$, antebraço e punho em neutro, segundo recomendação da Sociedade Americana dos Terapeutas da Mão[5,6]. Foi utilizada a empunhadura do dinamômetro na posição 3[7].

Os jogos selecionados para o fortalecimento no videogame foram: 1) monkeys - para preensão grossa, 2)Hit Walls- para pinça polpa-polpa, 3)Package- para pinça polpa-lateral, 4) Skateboard - para pinça polpatrípode.

O protocolo de fortalecimento foi realizado no membro lesado, e seguindo os regimes isotônicos da técnica de Oxford, sendo a primeira série com $100 \%$, a segunda série com $75 \%$ e a terceira série com $50 \%$ da carga do 10RM. Foram realizadas 3 séries de 10 
repetições mantendo a isometria de 5 segundos e o repouso de 5 segundos em cada repetição, totalizando 2 minutos de treinamento e repouso de 2 minutos entre as séries. Esse protocolo foi adaptado ao Protocolo de Oxford desenvolvidos por Zinovieff(1951).

Foram realizadas 2 sessões semanais, com duração de 40 minutos, totalizando 17 sessões de fortalecimento. Para análise dos dados foram considerados os valores pré e pós fortalecimento.

\section{Resultados}

Após 17 sessões de fortalecimento verificou-se um aumento das forças de preensão palmar e pinças em ambos os membros, lesado e não lesado, como demonstra a Tabela 2.

No membro lesado, o ganho de força foi 4,5 vezesmaior em relação a avaliação pré fortalecimento para preensão palmar. E para as pinças o ganho foi de 1,6 vezes para pinça polpa-polpa;1,3 vezes para pinça polpa-lateral e 1,2 vezes para pinça polpa- trípode.

O membro não lesado (que não recebeu treinamento) apresentou também um aumento da força de 1,2 vezes maior em relação a avaliação pré fortalecimento para preensão palmar; 1,4 vezes para pinça polpa-polpa; 1,1 vezes para pinça polpa-lateral e 1,2 para pinça polpatrípode.

Tabela 2: Forças de preensão e pinça pré e pós fortalecimento

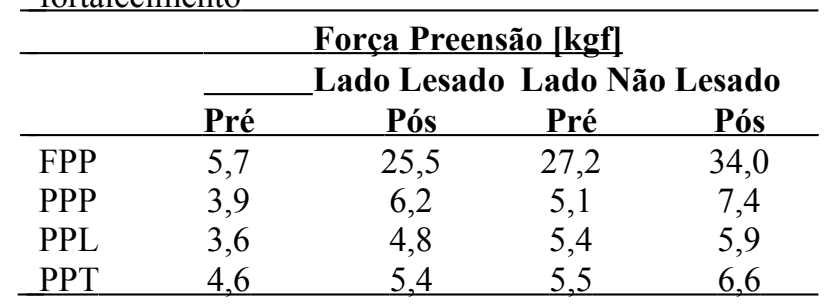

$\mathrm{FPP}=$ força de preensão palmar; PPP $=$ força pinça polpa-polpa; PPL = força pinça polpa-lateral; $\mathrm{PPT}=$ força pinça polpa-trípode; Kgf = quilograma força.

Quanto à avaliação funcional o paciente apresentou uma redução da incapacidade, determinada pelos escores dos questionários funcionais DASH e PRWE (Tabela 3).

Tabela 3: Valores das avaliações funcionais pré e pós a realização do fortalecimento

\begin{tabular}{lcc}
\hline & Pré & Pós \\
\hline DASH & $45 \%$ & $6,6 \%$ \\
PRWE & $30 \%$ & $8,5 \%$ \\
\hline
\end{tabular}

DASH $=$ Disability of the Arm, Shoulder and Hand; PRWE $=$ Patient Rated Wrist Evaluation .

\section{Discussão}

Esse estudo teve como objetivo analisar o efeito do uso do videogame $E$ - $L i n k$ no fortalecimento dos músculos da preensão e das pinças em um paciente com fratura complexa de punho.
O protocolo de Oxford foi escolhido para esse estudo, por demonstrar maior eficácia para os músculos da mão por ter como princípio, a redução da resistência a medida que o músculo fadiga e por apresentar melhores resultados quando comparado ao protocolo Delorme[9].

Os resultados obtidos nesse estudo demonstraram um aumento da força de preensão palmar e das pinças no membro lesado após a realização de um protocolo de fortalecimento por meio do videogame utilizando a técnica de Oxford, e uma redução da incapacidade dos membros superiores.

Para esse paciente podemos dizer que ele apresentou uma recuperação completa da força de preensão do membro lesado, pois segundo Perteson et al. (1989) foi constatado que o membro não dominante tem uma tendência a apresentar de 5 a $10 \%$ a menos de força em relação ao lado dominante. Com base nos resultados, o paciente obteve um aumento de $92,72 \%$ de força do membro lesado quando comparado a primeira avaliação da força de preensão do membro não lesado.

Dentre outros fatores que podem ter influenciado no aumento da força de preensão e das pinças destaca-se a utilização de ambientes virtuais. Em estudo realizado por Rostami et al. (2015) foram encontrados vários pontos positivos para utilização do videogame E-link na reabilitação do membro superior em pacientes com paralisia cerebral. Por meio da utilização de jogos foi possível comprovar a eficácia desse equipamento para o ganho da função da mão, que incluem aumento velocidade, força, amplitude de movimento e acurácia exigida nos jogos.

Em relação ao aumento da força do membro lesado, segundo Moritani (1992), Carroll et al. (2002), Maior e Alves (2003), isso ocorre pois nas fases iniciais do treinamento o aumento de força se dá quase que exclusivamente em resposta às adaptações neurais ao treinamento resistido.

Nessa fase, os ganhos da força podem ser conseguidos pelo aumento do recrutamento de unidades motoras do músculo envolvido no movimento, devido ao aprimoramento da ativação neural. Porém em relação a morfologia existe pouca influência da hipertrofia muscular no ganho de força, e não são observadas mudanças estruturais do tamanho dos músculos[14,15].

Também foi observado um aumento da força de preensão e das pinças no membro que não realizou o fortalecimento (não lesado). Embora seja claro que existe um aumento da força do lado contralateral ao lado treinado, ainda não existe na literatura um consenso sobre os mecanismos envolvidos por trás desse efeito[16]. Conceitualmente foram desenvolvidas duas classes diferentes de mecanismos que tentam explicar o aumento da força no lado não treinado. $\mathrm{O}$ primeiro teoriza que o treinamento de força unilateral poderia causar um "spillover" na condução neural para o lado não treinado, induzindo adaptações no sistema de controle. E o segundo mecanismo estaria relacionado as adaptações neuromusculares provocadas no sistema de controle do membro treinado que poderiam ser acessados pelo lado não treinado. Foi estabelecido que o 
ganho de força contralateral não resulta em alterações na morfologia muscular[17].Entretanto acreditamos que no caso de trauma no membro superior, o paciente passa a utilizar apenas o membro não lesado para realizar todas as atividades durante o período de recuperação da mão lesada e essa atividade uni manual possa ter esse efeito no ganho da força.

Em relação aos questionários de avaliação funcional, estes são importantes pois fornecem ao terapeuta informações sobre o nível de função do paciente. Para esses pacientes, a realização dos questionário contribuem para tomada de decisões no processo de reabilitação [18].

Mediante o exposto, fica claro a necessidade do emprego de novas estratégias e combinações de terapia que potencializem a recuperação da funcionalidade, da amplitude de movimento e da força muscular, proporcionando maior independência funcional $\mathrm{e}$ melhor qualidade de vida para esses pacientes.

\section{Conclusão}

Conclui-se que o videogame foi uma ferramenta eficaz no aumento das forças de preensão e pinça e na redução da incapacidade funcional para este paciente.

\section{Agradecimentos}

Os autores agradecem ao LABCOM por ceder espaço e equipamentos, a Coordenação de Aperfeiçoamento de Pessoal de Nível Superior (CAPES) pelo apoio financeiro e ao voluntário que aceitou participar desse estudo.

\section{Referências}

[1] SOUZA MAP, DE ASSIS CABRAL LH, SAMPAIO RF, MANCINI MC. Acidentes de trabalho envolvendo mãos: casos atendidos em um serviço de reabilitação. Fisioterapiae Pesquisa. 2008;15(1):64-71.

[2] BRASIL. Ministério da Previdência e Assistência Social. Anuário estatístico da Previdência Social 2006. 2010.

[3] LIMA APT, RIBEIRO IA, COIMBRA LM de C, DOS SANTOS MRN, DE ANDRADE EN. Mecanoterapia e fortalecimento muscular: um embasamento seguro para um tratamento eficaz. Rev Saúde Com. 2006;2(2):143-52.

[4] ALBUQUERQUE EC, SCALABRIN EE. The Use of the Computer in Neuropsychological Rehabilitation Programs. Psicol Argum. 2007;25(50):269-275.

[5] FESS EE. Grip Strength. 2nd ed. Chicago: American Society of Hand Therapists; 1992.

[6] FESS EE, MORAN CA. Clinical assessment recommendations. Philadelphia: American Society of
Hand Therapists; 1981.

[7] FERNANDES LFRM, BERTONCELLO D, PINHEIRO NM, DRUMOND LC. Correlações entre força de preensão manual e variáveis antropométricas da mão de jovens adultos. Fisioter e Pesqui. 2011;18(2):151-156.

[8] ZINOVIEFF A. Heavy-resistance exercises the "Oxford technique." The British journal of physical medicine: including its application to industry. 1951;14(6):129-32.

[9] FERNANDES L, ARAÚJO MS, MATHEUS JPC, MEDALHA CC, SHIMANO AC, PEREIRA GA. Comparação de dois protocolos de fortalecimento para preensão palmar. Rev Bras Fisioter. 2003;7(1):17-23.

[10] PETERSEN P, PETRICK M, CONNOR H, CONKLIN D. Grip strength and hand dominance: challenging the $10 \%$ rule. Am J Occup Ther. 1989;43(7):444-447.

[11] ROSTAMI H, NEJAD S, ARASTOO A. Effects of Practice in Virtual Environment on Upper Limb Function of Children with Spastic Hemiparetic Cerebral Palsy. Iran Neurology. 2015;

[12] MORITANI T. Neuromuscular adaptations during the acquisition of muscle strength, power and motor tasks. Journal of Biomechanics. 1993;26:95-107.

[13] CARROLL T, RIEK S, CARSON R. Neural adaptations to resistance training. Sports medicine. 2001;31(12):829-40.

[14] MAIOR A, ALVES A. A contribuição dos fatores neurais em fases iniciais do treinamento de força muscular: uma revisão bibliográfica. Motriz. 2003;9(3):161-8.

[15] HICKSON R, HIDAKA K, FOSTER C. Skeletal muscle fiber type, resistance training, and strengthrelated performance. Medicine and science in sports and exercise. 1994;26(5):593-8.

[16] MUNN J. Contralateral effects of unilateral resistance training: a meta-analysis. J Appl Physiol. 2004 May 1;96(5):1861-6.

[17] CARROLL TJ, HERBERT RD, MUNN J, LEE M, GANDEVIA SC. Contralateral effects of unilateral strength training: evidence and possible mechanisms. J Appl Physiol. 2006 Jul 27;101(5):1514-22.

[18] MACDERMID JC, TOTTENHAM V. Responsiveness of the disability of the arm, shoulder, and hand (DASH) and patient-rated wrist/hand evaluation (PRWHE) in evaluating change after hand therapy. J Hand Ther. 2004 Jan;17(1):18-23. 